\title{
Afectaciones psicológicas que experimentan adolescentes afrodescendientes con padres embarcados
}

Marina Danila Mc coy ${ }^{1}$ Lyssette Newball Crisanto ${ }^{2}$ Neidy Gutiérrez Soza ${ }^{3}$

\section{Resumen}

Esta investigación ha abordado las afectaciones psicológicas que experimentan adolescentes afrodescendientes con padres embarcados. Con una metodología tanto cuantitativa como cualitativa, con 70 estudiantes de primero a quinto año del Colegio Moravo de la ciudad de Bluefields, mediante la aplicación de encuestas y grupos focales.

Entre los principales resultados, se puede mencionar que desde la perspectiva de los adolescentes que les afecta la ausencia de sus padres en los siguientes niveles: Mucho $40 \%$, poco en un $37 \%$ y nada en $23 \%$. Sin embargo, a pesar de la distancia, el $72 \%$ plantea que es buena la relación con sus progenitores, el $21 \%$ que es regular y el $7 \%$ es indiferente.

También están conscientes de los sacrificios que hacen sus padres para mejorar su nivel de vida. Valoran que el apoyo percibido de forma económica que corresponde a $54 \%$, emocional $44 \%$ y $1 \%$ espiritual; solamente el $1 \%$ manifestó no sentirse apoyado.

En relación al rendimiento académico, el $50 \%$ alto, el $46 \%$ medio y $4 \%$ bajo. Estos datos coinciden con las valoraciones de los docentes. Para enfrentar la ausencia de los padres, realizan diferentes actividades, el $49 \%$ deportiva, $4 \%$ en grupos religiosos y $47 \%$ en actividades sociales.

En relación a las conductas adquiridas, manifestaron estar aislados, rebeldes, indisciplinados, distraídos, independientes. El $86 \%$ se identifican con una de estas conductas mientras que el $14 \%$ dicen no manifestar ninguna de ellas.

Palabras clave: Afrodescendientes; adolescentes; afectaciones psicológicas; migración.

1 Licenciada en Psicología en Contexto Multiculturales y Psicóloga del Centro de Atención Psico Social (CAPS) de Bluefields. Mccoy. marina@yahoo.es

2 Licenciada en Psicología en Contexto Multiculturales Psicóloga en MAPS - FADCANIC de Bluefields. Gp200932@yahoo.com

3 Master en Antropología Social con mención en Desarrollo Humano, Tutora de la Investigación. neidygs@yahoo.com 


\section{Summary}

This research has addressed the psychological effects experienced by Afrodescendant adolescents with shipped-out parents. It's focused on a quantitative and qualitative methodology, with 70 students from first to fifth year of the Moravian School in the city of Bluefields, by conducting surveys and focus groups.

Among the main results, it can be mentioned that from the perspective of adolescents that are affected by the absence of their parents we find the following levels: A lot $40 \%$, little bit $37 \%$ and $23 \%$ nothing. However, despite the distance, $72 \%$ of the teenagers suggests that the relationship with their parents is good, $21 \%$ says is regular and $7 \%$ says it is indifferent.

They are also aware of the sacrifices that their parents do in order to improve their standard of living. Their appreciation is that the economic support perceived from their parents corresponds to $54 \%, 44 \%$ emotional and spiritual 1\%; only $1 \%$ expressed not to feel supported.

With regard to the academic performance, the $50 \%$ is high, $46 \%$ medium and $4 \%$ is low. These data are consistent with teachers' evaluations. To face the absence of parents they perform different activities: sports $49 \%, 4 \%$ in religious groups and $47 \%$ in social activities.

In relation to the acquired behaviors, they expressed to be isolated, rebellious, undisciplined, distracted and independent. 86\% identify themselves with one of these behaviors while $14 \%$ say they don't present any of them.

Keywords: Afrodescendants; adolescents; psychological effects; migration.

\section{Introducción}

El tema que aborda esta investigación son las afectaciones psicológicas que experimentan adolescentes afrodescendientes con padres embarcados, "Ship - Out" y cómo esta situación influye en su comportamiento y su rendimiento escolar.

En muchas ocasiones, durante el proceso migratorio los niños, niñas y jóvenes pueden afrontar sentimientos de pérdida y/o desarraigo, cambios en la identidad personal y de su entorno (Save the Children; 2005).Para muchas personas de las Regiones autónomas de la Costa Caribe la migración es una opción para buscar empleo fuera del país y así mejorar las condiciones de vida de sus familiares. 
La población afrodescendiente es una de las etnias de la región que más opta por migrar en busca de trabajo en barcos de cruceros en donde se ausentan por largos períodos de tiempo y los hijos e hijas quedan solos o al cuido de sus familiares, causando con su partida una desintegración familiar y desequilibrio emocional en sus hijos, siendo afectados por la carencia física de sus padres.

Los resultados permiten conocer los sentimientos de los adolescentes cuando sus padres se separan de ellos en busca de mejores oportunidades de trabajo para sustentarlos económicamente. De igual forma, se discuten las estrategias que utilizan para afrontar la ausencia de sus padres, por lo que, el presente documento, es un insumo de reflexión para padres y madres que optan por la migración. También ayuda a profesores y a sus tutores a comprender las afectaciones psicológicas que presentan los adolescentes con padres embarcados.

\section{Revisión de literatura}

Afectividad es la esfera de los sentimientos y de las emociones que interactúan con la esfera motriz y con la intelectual, de la que se distingue sólo abstractamente (Galimberti, 2002). Afectación psicológica es para este estudio, una alteración de la salud mental, expresada mediante sentimientos y emociones que inciden desfavorablemente en el comportamiento y rendimiento académico de los adolescentes con padres embarcados.

Sentimiento y emociones, según Duque y Vieco (2007), el primero de estos, corresponden a las reacciones que percibimos por medio de los sentidos y dan forma a nuestras reacciones frente a lo que percibimos en el futuro. Los sentimientos nos dicen si lo que experimentamos es amenazador, doloroso, triste o regocijante.

La desintegración familiar, puede acarrear problemas psicológicos a los niños y adolescentes, que pueden traducirse en un bajo rendimiento escolar, alcoholismo, drogadicción, pandillerismo, intentos de suicidio, entre otras patologías sociales. Situaciones que deben ser tomadas en cuenta, cuando se estudia el fenómeno migratorio (Chevez, 2012).

La migración de los padres influye en el crecimiento y maduración de los hijos. En su mayoría, asumen responsabilidades que no son propias de su edad y experimentan los cambios fundamentales de su vida alejados de los progenitores o, al menos, de uno de ellos. La migración es un proceso de cambio, implica enfrentarse a nuevas relaciones y experiencias, asumir nuevas responsabilidades, adquirir nuevas destrezas y habilidades (Fe y Alegría, 2010).

La adolescencia es un período crítico del ciclo de la vida, y como todos los períodos críticos está llenos de cambios (cambios del cuerpo, de la forma de pensar, 


\section{CIENCIAS SOCIALES}

emocionales), de inseguridades (ansiedad sobre su futuro, sobre las vicisitudes de su desarrollo), desequilibrios entre aspiraciones y oportunidades (en el terreno afectivo, sexual, académico, laboral) y presiones de los padres, de sus coetáneos, de la sociedad de consumo (Ruiz, 2004).

Desarrollo psicosocial, según Erikson citado por Clonninger S. (2002), en esta etapa de identidad versus confusión de la identidad (Desde los 12 hasta los 18 años aproximadamente). Se experimenta búsqueda de identidad y una crisis de identidad, que reavivará los conflictos en cada una de las etapas anteriores; los padres de los adolescentes se verán enfrentando situaciones nuevas que serán un nuevo reto para su misión orientadora.

Teoría del Apego según Fonagy y Mendiola (2005), parte de la propensión de los seres humanos a formar fuertes lazos afectivos con personas determinadas. Por apego se entiende el lazo afectivo que se forma entre el niño y su figura materna, este vínculo se infiere de una tendencia estable a lo largo del tiempo de buscar proximidad y contacto con esta figura específica. En el adulto el apego se activa cuando hay estrés, enfermedad y en momentos difíciles en donde la persona tiende a sentirse muy vulnerable.

Comportamiento y conductas: son acciones observables y procesos mentales de personas y animales (Spencer, 1987). Se refiere a las acciones y reacciones del sujeto ante el medio. Se entiende por conducta humana como todo aquello que una persona hace, piensa o dice en relación al ambiente que le rodea, actual o pasado, físico o social constituyendo una adaptación perfecta al entorno.

Para un niño la unidad de su familia es básica. Al romperse la unidad familiar, se ponen en tela de juicio todos los afectos; ausencia de los padres desencadena problemas en los niños de índole: emocional, afectiva, educativa, salud y social. En este sentido, según Kubler Ross (1975), cuando hay separación de la familia los niños experimentan un duelo, el cual se pasa por cinco etapas que se presentan en su totalidad para el niño cuando se rompe el vínculo familiar, las cuales son: negación, ira, negociación, depresión y aceptación".

En cuanto a las estrategias de afrontamiento, Lazarus y Folkman (1984) definen al afrontamiento como "aquellos esfuerzos cognitivos y conductuales constantemente cambiantes que se desarrollan para manejar las demandas específicas externas y/o internas que son evaluadas como excedentes o desbordantes de los recursos del individuo"

El afrontamiento es definido "como un esfuerzo para reducir o mitigar los efectos aversivos del estrés, esfuerzos que pueden ser psicológicos o conductuales. Las estrategias de afrontamiento más usadas por las adolescentes mujeres se asocia con 
búsqueda de apoyo social. Los adolescentes varones recurren a estrategias activas, haciendo deportes y manteniéndose activos (Cox, 2007).

\section{Materiales y métodos}

El tipo de investigación que se realizó es mixto, porque según Sampieri (2006) estas investigaciones tienen tanto un enfoque cuantitativo como cualitativo. La población de estudio correspondió a un total de 78 estudiantes, es decir, todos aquellos jóvenes afrodescendientes de 1er año a 5to, con padres embarcados del Colegio Moravo de Secundaria. Sin embargo, solamente 70 cedieron su consentimiento para participar en el estudio, representado de esta forma un muestra del $89.74 \%$.

Para el enfoque cualitativo se seleccionó al azar a 10 estudiantes de los 70 estudiantes encuestados para formar un grupo focal y se entrevistó a 6 docentes. Se excluyeron a estudiantes que no se identifican con la etnia afrodescendientes, a los que no tienen a sus padres embarcados y a los que no desean participar de manera voluntaria.

Como consideraciones éticas se les comunicó a los estudiantes el objetivo del estudio solicitando su participación voluntaria y que su información será de forma confidencial, segura y sin alteración. Se desarrolló total transparencia sin dañar la integridad de las personas y se les informó que sus respuestas son de mucha importancia, que deben de ser honestos sin necesidad de temor ya que su información (identidad) no será mostrada a nadie.

El procesamiento de la información cuantitativa se realizó mediante el programa SPSS. La información cualitativa se procesó y analizó, mediante matrices diseñadas a partir de las variables y descriptores del estudio.

\section{Resultados y discusión}

La presente investigación se realizó con el propósito de conocer las afectaciones psicológicas de los adolescentes (mujeres y hombres) cuyos padres trabajan embarcados. Los resultados y discusión de esta investigación se han ordenado de tal manera que se describen al inicio las principales características de los jóvenes y sus familias. Posteriormente, se describen los sentimientos, emociones y comportamientos que estos desarrollan ante la ausencia de sus padres, así como el efecto en el rendimiento académico. Finalmente, se describen las estrategias que estos jóvenes desarrollan para enfrentar dicha situación. 
En relación a las características generales de estos adolescentes, se encuestaron a 70 estudiantes. En lo que respecta al género, el $36 \%$ de la población encuestada son varones y el $64 \%$ son mujeres.

Las edades de la población se presentan en la figura No. 1, en donde la mayoría de los adolescentes que participaron en el estudio oscilan las edades correspondientes a los 13, 15, 1617 años.

\section{Edades de los Adolescentes}

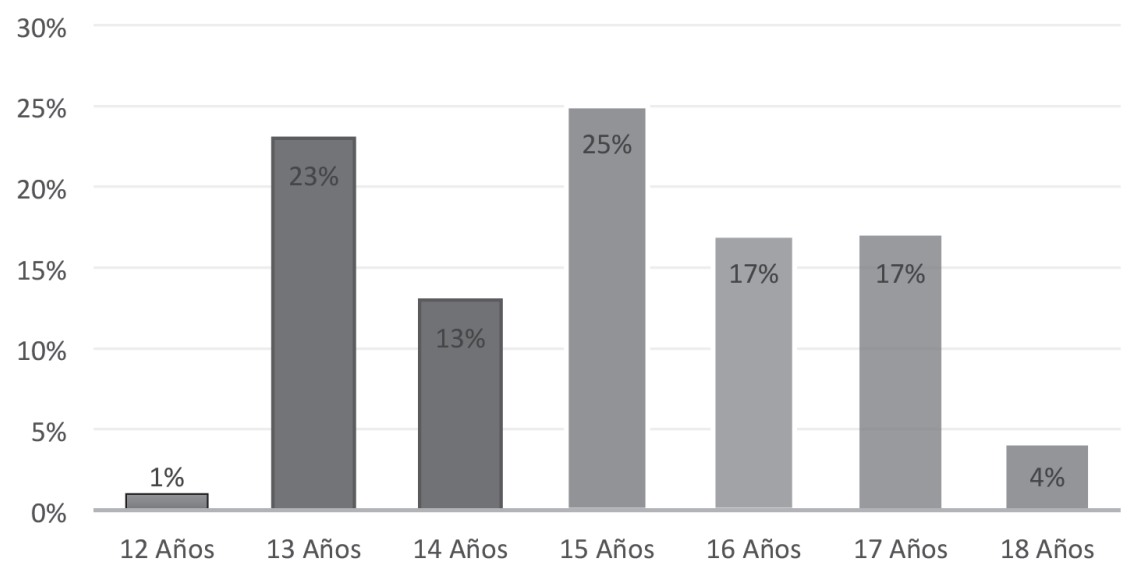

Figura No. 1. Edades de los adolescentes

\section{Sentimientos, emociones y comportamiento de los adolescentes}

Desde la perspectiva de los adolescentes plantean que la ausencia de padre/madre les afecta en los diferentes niveles: mucho con el $40 \%$, el $37 \%$ dicen que les afecta poco mientras un $23 \%$ opinan que en nada les afecta que su padre/madre no estén presentes.

Esta categorización se elaboró, tomando en consideración los aportes de Chevez (2012) en donde se plantea que la separación de hijos e hijas de sus padres, puede acarrear problemas psicológicos en los niños y adolescentes. En cambio, Salazar (2002), manifiesta que las penurias emocionales que implica la separación, no necesariamente repercute en el bienestar emocional de los hijos.

Kubler Ross (1975), plantea que la ausencia de los padres influye significativamente en el desarrollo emocional de los hijos e hijas, debido a que se rompe la unidad familiar y se pone en tela de juicio todos los afectos de índole emocional, afectiva, social, educativa. Esta investigación obtuvo resultados similares a la investigación de Ross; mediante los instrumentos aplicados, encuesta y grupo focal en las cuales 
los/as adolescentes plantearon que necesitan el afecto de sus padres en diferentes momentos de su vida cotidiana.

Al preguntarles si la ausencia de sus padres les afecta, estos plantearon lo siguiente: Sí, me ha afectado mucho, porque a veces lo necesito a mi lado y soy su niña muy querida; extraño sus palabras, sus abrazos, extraño su mirada.

Si porque solo ellos me daban la atención, amor y cariño que yo necesito.

Estudiante del colegio Moravo.

En relación al crecimiento de los/as hijos/as, los adolescentes han planteado que la ausencia de sus padres, han incidido en su desarrollo. Es decir, en la mayoría de los casos, sienten que sus padres no han estado en los mejores momentos de su niñez y adolescencia:

Si porque solo lo veo tres meses al año ya que trabaja siete meses al año, no me ha visto crecer.

Cuando mi mamá no está siento que tengo mucha responsabilidad, porque tengo que cuidar de mis hermanitos menores y a la misma vez estudiar.

Estudiantes del colegio Moravo

La migración de los padres influye en el crecimiento y maduración de los hijos. En su mayoría, asumen responsabilidades que no son propias de su edad (Fe y Alegría, 2010). Al embarcarse el padre o la madre algunos adolescentes por ser los hermanos mayores quedan al cuido de sus hermanos menores, dejando de disfrutar en muchas ocasiones de algunas actividades de diversión propia de sus edades.

El 23\% manifiesta que no les afecta en nada que sus progenitores no estén presentes, por ende, se considera que los vínculos afectivos que se desarrolla desde la niñez con los progenitores se perdieron al paso del tiempo y la distancia. Es decir, cuando los padres pasan trabajando fuera del hogar (en barcos cruceros hasta 10 años), se rompe de esta forma la unión familiar dando paso a la frialdad en la comunicación.

En cuanto a la relación de padres e hijos la mayoría de los adolescentes manifiestan que mantienen una buena relación con sus padres a pesar que están lejos, representando así el 72\%. Otros adolescentes dicen que su relación es regular con el $21 \%$ y otros dicen que es indiferente la relación que tienen con sus padres, esto es el $7 \%$. 


\section{Relación que tiene los adolescentes con sus padres}

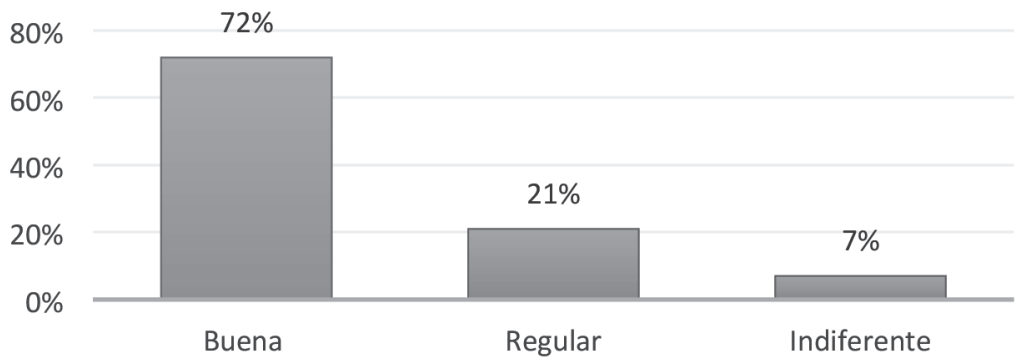

Figura No. 2. Relación que tienen los adolescentes con sus padres

La mayoría de los adolescentes plantearon que aunque sus padres estén lejos, la relación con ellos es positiva, manteniendo una buena comunicación y estrecha relación que resaltan como fundamental para la unión de la familia a pesar de la distancia. Sin embargo, es importante mencionar que los resultados de esta investigación no coinciden con lo afirmado por Fe y Alegría (2010) que señala lo siguiente: pese a los esfuerzos que realizan las familias y sobre todo las migrantes a través de la comunicación permanente, se están produciendo cambios negativos en la relación con la familia que se expresan en la mayoría de los/as hijos/as adolescentes con comportamiento rebelde.

Ante esto, unos adolescentes plantean lo siguiente: "La relación no cambia porque mantenemos una buena comunicación" [...] "Mi relación con ellos es buena porque les tengo confianza"

Mientras que otros dicen tener una relación indiferente porque sus padres están embarcados desde que ellos nacieron y no tienen ese vínculo amoroso con ellos;

Encontramos similitudes con las investigaciones realizada por Chevez (2012) los hijos pequeños establecen una distancia emocional con el padre ya que no han tenido oportunidad de establecer lazos afectivos debido a su ausencia física, mientras que los hijos adolescentes pueden llegar a resentir la autoridad paterna cuando a través de la distancia, este, trata de controlar o incidir en la conducta de los jóvenes.

“Mi papa se fue embarcado desde que nací, por eso obedezco más a mamá que a papá”. 
Con respecto al comportamiento o conductas adquiridas por la ausencia de los padres se ilustran en la figura No. 3 .

\section{Conductas Adquiridas}

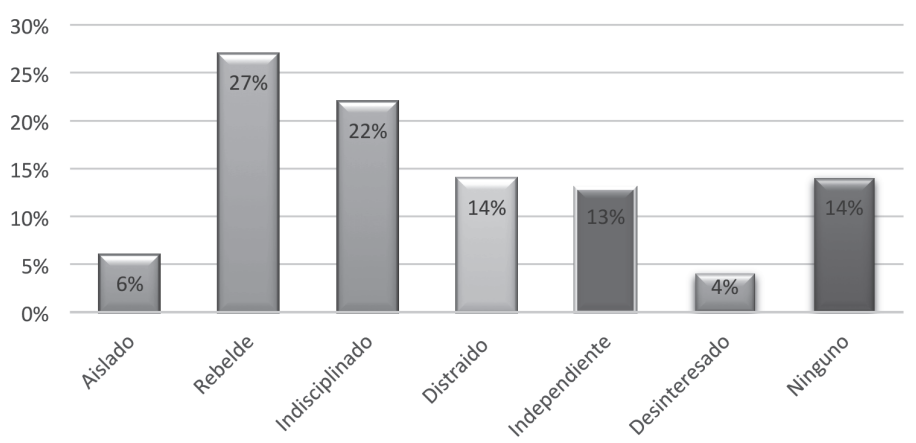

Figura No. 3. Conductas adquiridas

Basado en lo anterior, los autores en nuestro marco teórico, tienen distintos planteamiento acerca de que la ausencia de los padres afecta negativamente el comportamiento. Por ende, se considera que la ausencia de los padres incide en el estado emocional del adolescente, pero sus comportamientos, no responden necesariamente a dicha separación, sino más bien a los diferentes cambios físicos y psicológicos, propios de la etapa de adolescencia.

Retomando la teoría psicosocial de Erikson sobre la etapa búsqueda de Identidad versus confusión de la identidad, según la referencia de Clonninger, S. (2002), se hace referencia a que los adolescentes experimentan búsqueda y una crisis de identidad; las cuales son características del adolescente por la etapa en la que se encuentra y por ende en esta investigación no se relaciona a la separación que tienen con sus padres que trabajan embarcados.

Bandura (1982), en la teoría del aprendizaje social sostiene que las personas aprenden tanto a partir de las observaciones del comportamiento de otros como partir de su propia experiencia. Lo anterior, se plantea, desde la valoración de los docentes, quienes expresaron que los adolescentes, cuyos padres están embarcados, no presentan problemas complejos de comportamiento de indisciplina, salvo algunos casos muy excepcionales.

Algunos adolescentes manifiestan que no cambian su comportamiento, actúan igual estando sus padres presentes o ausentes, por ende su conducta no varía cuando vienen los padres de vacaciones, sin embargo esto no significa que no presenten conductas de rebeldía, indisciplina entre otros, pero estas no son adquiridas necesariamente por la falta de presencia de sus padres en sus vidas. Estas conductas pueden ser parte del proceso de transición del adolecente, también depende de los diferentes 
estilos de crianza que estos reciban de su padre, madre o encargado, manifestando comportamientos negativos o positivos.

"Sí, soy rebelde no es por lo que no esté mi padre, sino porque siempre he sido así"

Estudiante del Colegio Moravo

Por su lado, los docentes que participaron en la entrevista de esta investigación al referirse al comportamiento de los/as adolescentes señalaron lo siguiente:

"Bueno, la mayoría de estos adolescentes tienen un comportamiento aceptable"

Docente del colegio Moravo.

Algunas de las conductas varían en dependencia del estilo de crianza (permisivo, autoritario, indiferente, autoritativo) que reciben en el hogar, en donde muchas veces el padre/madre ejerce mayor autoridad y disciplina sobre las hijas e hijos, al estar estos ausentes, se resisten a la autoridad que ejercen sobre ellos los que quedan a cargo del adolescente.

\section{Rendimiento académico de los adolescentes con padres embarcados}

Con respecto al efecto que tiene la migración de los padres en el rendimiento académico de los hijos; los adolescentes expresaban que no les afectaba mucho que sus padres no estuvieran presentes físicamente en las diferentes etapas de su educación. Por tanto, en la figura 4, se presentan los resultados de la encuesta según el rendimiento académico.

\section{Rendimiento Académico}

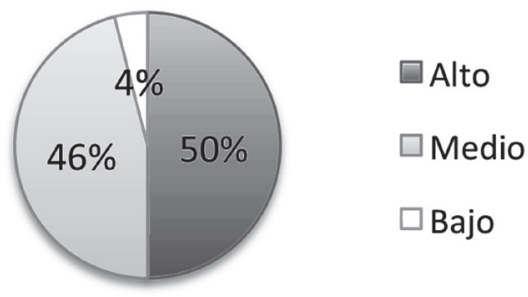

Figura No. 4. Rendimiento académico de los estudiantes

$\mathrm{Al}$ analizar los resultados obtenidos en este estudio revelan que aunque el padre/ madre se encuentra trabajando fuera del país, las hijas e hijos muestran interés de superarse mediante sus estudios, desde la perspectiva del estudiante los resultados obtenido son: un 50\% de los estudiantes tiene un nivel académico alto y un $46 \%$ tiene un rendimiento académico medio y un $4 \%$ es bajo. 
En entrevistas realizadas a maestros expresaban que los estudiantes con padres embarcados en su rendimiento académico no muestran diferencia en relación a los estudiantes con padres no embarcados.

Ante esto, los profesores expresan:

"Los hijos de padres embarcados tienen buenas notas en cambio otros presentan notas bajas"

Docentes del Colegio Moravo.

Fe y Alegría (2010), plantea que las hijas e hijos ven la migración de su padre o madre como un estímulo para su superación en la escuela y que estos perciben con mucha claridad que los motivos de la migración de sus padres son económicos y que lo hacen por ellos.

\section{Estrategias de afrontamiento de la ausencia de los padres}

Con relación a las estrategias que utilizan las hijas e hijos con padres embarcados (ship-out) esta investigación obtuvo los siguientes resultados:

\section{Estrategias de afrontamiento de los adolescentes ante la ausencia de sus padres}

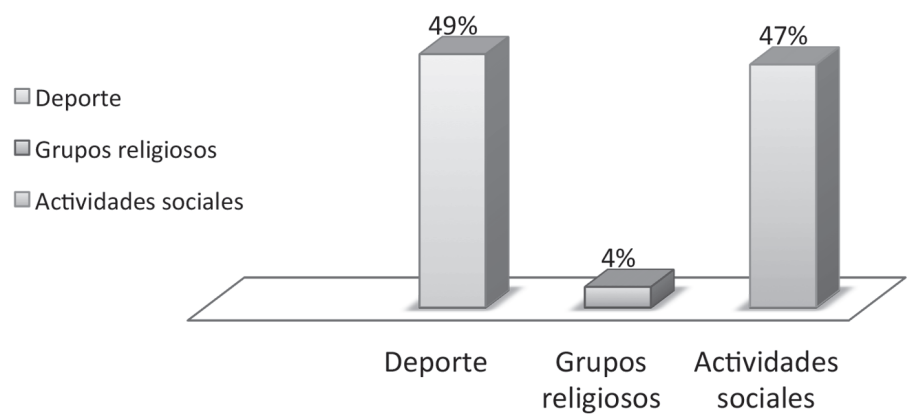

Figura No. 5. Estrategias de afrontamiento de los adolescentes ante la ausencia de sus padres

Lo anterior, corresponde a un $49 \%$ que se distrae en actividades deportivas y un $4 \%$ se dedica a grupos religiosos y un $47 \%$ en actividades sociales tales como: navegación en internet, Skype, Facebook, centros de diversiones, ver juegos deportivos (estadio, gimnasio del colegio moravo, multiuso), parque etc. 
Según Ruiz (2004), es importante ayudar a los adolescentes a aprender los múltiples mecanismos psicológicos de adaptación y las estrategias de afrontamiento que les van a ayudar a vivir, a relacionarse de forma satisfactoria y a desarrollar todas sus potencialidades.

Los adolescentes con padres embarcados han utilizado algunas estrategias de afrontamiento para proteger su estado emocional ante la ausencia de sus padres cuando estos tienen que regresar a trabajara los barcos cruceros.

Los resultados de la presente investigación difieren de la hipótesis planteada inicialmente, en donde se consideraba que los adolescentes afrodescendientes con padres embarcados son afectados psicológicamente por la ausencia de sus padres y que esto incidía en sus comportamientos y en sus rendimientos académicos. Sin embargo, los resultados señalaron que los adolescentes manifiestan sentimientos de soledad, tristeza, los cuales no se consideran que lleguen a un estado patológico.

\section{Conclusiones}

El presente estudio culminó permitiendo obtener información sobre las afectaciones psicológicas que experimentan los adolescentes con padres que trabajan en barcos cruceros, por ende a continuación señalamos las siguientes conclusiones:

- Algunas afectaciones psicológicas que sufren los adolescentes (mujeres y hombres) con padres embarcados son la soledad, tristeza y falta de afecto.

- La ausencia de los padres y madres de los adolescentes no tiene gran impacto en el rendimiento académico, por lo que han mantenido un buen desempeño escolar.

- Para enfrentar la ausencia de sus padres los adolescentes han recurrido a diferentes estrategias de afrontamiento (actividades deportivas y sociales) para disminuir los sentimientos de soledad, tristeza y abandono físico, estos tratan de sobrellevar esta situación y acostumbrarse ella.

- Comportamientos como la distracción, la indisciplina, rebeldía no se podría decir que tiene una relación directa con la ausencia de los padres. Sino más bien, a los múltiples factores que implica este proceso de transformación físico y emocional en los adolescentes.

- Se concluye que la ausencia del padre o la madre embarcado no siempre es desfavorable, para los hijos que quedan en el país de origen, la mayor parte de las veces busca mejorar la calidad de vida y la economía de sus hijos y familias; sin embargo, podemos señalar que si no se realiza de manera planificada y ordenada, puede causar mayores consecuencia al tejido familiar y social de los adolescentes. 


\section{Lista de referencias}

Bandura, A. (1982). Teoría del aprendizaje social. Madrid: Espasa-Calpe.

Chevez, J. S. (2012). Los efectos Psicológicos de la migración parental sobre la autoestima de los adolescentes en zonas de alta tradición migratoria. (Tesis, Universidad Nacional Autónoma de México). Recuperado el 10 de Febrero del 2013 de http://www. citeach.org/web/wp-content/uploads/2012/og/ART4migracionparental.pdf

Clonninger, S. (2002). Teorías de la personalidad. Pearson Educación.

Duque, H \& Vieco, L. (2007). Conozca sus Sentimientos y Emociones. Recuperado el 26 de febrero de http://books.google.com.ni/books?id=2QdWp1yIfYYC\&printse $\mathrm{c}=$ frontcover\&dq=emociones+y+sentimientos\&hl=es\&sa=X\&ei=RSctUd2UA5 CNoQG2-4CwDQ\&sqi=2\&ved=oCDoQ6AEwAg

Fe y Alegría (2010). Madurar sin padres: Los efectos de la migración de los progenitores en el desempeño escolar de los niños y adolescentes en Bolivia. Recuperado el 10 de Octubre de http://www.entreculturas.org/files/documentos/estudios_e_informes/Informe\%2oBolivia_Madurar\%2osin\%2opadres.pdf?download

Fonagy P. , Mendiola, R. (2005). Teoría del apego y psicoanálisis. Revista Aperturas Psicoanalisticas $\mathrm{N}^{\circ}$ 20. Disponible: http://www.aperturas.org/articulos. php?id=0000539\&a=-Teoria-del-apego-y-psicoanalisis

Galimberti, U. (2002). Diccionario de la psicología. Siglo xxi editores, s, a de c.v. impreso en México Zepeda H F. 2003. Introducción a la psicología. PEARSON EDUCACION, México, S.A de C.V. ISBN: 970-26-0921-4. Segunda edición. Recuperado el o6 de marzo 2013 de http://books.google.com.ni/books?id=AfgrxAjmdFgC\&pg= $\mathrm{PT} 28 \& \mathrm{dq}=$ como+define + la + psicologia + wilhelm + wundt\&hl $=\mathrm{es} \& \mathrm{~cd}=9$

Kubler Ross E. (1975). Sobre la muerte y los moribundos. Ediciones Grijalbo Barcelona.

Lazarus, R. S. \&Folkman, S. (1984). Estrés y procesos cognitivos. Barcelona: Martínez Roca.

Ruiz, P. (2004). Promoviendo la adaptación saludable de nuestros adolescentes: proyecto de promoción de la salud mental para adolescentes y padres de adolescentes. Ministerio de Sanidad y consumo, Madrid, España. Recuperado el 14 de Febrero del 2013 de www.promensana.es/content/adolescencia-salud-mental.

Salazar P. R. (2002). The Care Crisis in the Philippines: Children and Transnational Families in the New Global Economy. In Global Women: Nannies, maids and Sex 
Workers in the New Economy, Edited by Barbara Ehrenreich and Arlie Russell Hochschild, New York, Metropolitan Books.

Sampieri R. (2006). Metodología de la investigación. McGraw- Hill/ Interamericana. México. Cuarta edición.

Save the Children (2005). Posicionamiento: niños y niñas afectados por los procesos Migratorios. Recuperado el 14 de Febrero del 2013 de http://www.savethechildren.es/docs/Ficheros/245/Poscionamiento_Migragiones.pdf

Spencer A. Rathus. (1987). Psychology. Library of congress cataloging- in Publication data. Thirdedition. 